Pacific Journal of Mathematic 


\title{
ORTHOGONAL HARMONIC POLYNOMIALS
}

\author{
P. R. GARABEDIAN
}

1. Introduction. In this paper we develop sets of harmonic polynomials in $x, y, z$ which are orthogonal over prolate and oblate spheroids. The orthogonality is taken in several different norms, each of which leads to the discussion of a partial differential equation by means of the kernel of the orthogonal system corresponding to that norm. The principal point of interest is that the orthogonality of the harmonic polynomials in question does not depend on the shape of the spheroids, but only on their size. More precisely, the polynomials depend only on the location of the foci of the ellipse generating the spheroid, and not on its eccentricity.

The importance of constructing these polynomials stems from the role which they play in the calculation of the kernel functions and Green's functions of the Laplace and biharmonic equations in a spheroid. One can compute from the kernels, in turn, the solution of the basic boundary-value problems for these equations. As a particular case, one arrives at formulas for the solution of the partial differential equation

$$
\frac{\partial^{2} f}{\partial \rho^{2}}+\frac{1}{\rho} \frac{\partial f}{\partial \rho}+\frac{\partial^{2} f}{\partial z^{2}}=0
$$

which arises in discussion of axially symmetric flow.

Results of the type presented here have occurred previously in the work of Zaremba [10], and are related to recent developments of Friedrichs $[3,4]$ and the author [5]. The polynomials investigated in this earlier work are in two independent real variables and yield formulas for solving the Laplace and biharmonic equations in two dimensions. Thus it is natural to suggest that the basic results generalize to $n$-dimensional space. In this connection, it is

Received September 21, 1951. The author wishes to express his thanks to Professor G. Szegö, who has given the first proof of the orthogonality of the polynomials introduced here, and who has shown a most friendly and encouraging interest in the questions related to them, for his collaboration in developing the results of this paper.

Pacific J. Math. 3 (1953), 585-603 
easily verified that a part of the theory carries over to arbitrary ellipsoids in three-dimensional space.

2. Notation and definitions. We shall make use of rectangular coordinates $x, y, z$, cylindrical coordinates $\rho, \phi, z$, and spherical coordinates $r, \theta, \phi$. Thus

$$
\begin{aligned}
x=\rho \cos \phi & =r \sin \theta \cos \phi, \\
y=\rho \sin \phi & =r \sin \theta \sin \phi, \\
z & =r \cos \theta .
\end{aligned}
$$

The Laplace integral formula

$$
P_{n}^{h}(\cos \theta)=\frac{(n+h) !}{\pi i^{h} n !} \int_{0}^{\pi}(\cos \theta+i \sin \theta \cos t)^{n} \cos h t d t
$$

for the Legendre polynomials $P_{n}(\cos \theta)=P_{n}^{0}(\cos \theta)$ and the associated Legendre functions $P_{n}^{h}(\cos \theta)$ is basic for our work. In terms of Laplace's integral we obtain the solid spherical harmonics in the form

$$
\begin{aligned}
& r^{n} P_{n}^{h}(\cos \theta) \cos h \phi=\frac{(n+h) !}{\pi i^{h} n !} \int_{0}^{\pi}(z+i \rho \cos t)^{n} \cos h \phi \cos h t d t, \\
& r^{n} P_{n}^{h}(\cos \theta) \sin h \phi=\frac{(n+h) !}{\pi i^{h} n !} \int_{0}^{\pi}(z+i \rho \cos t)^{n} \sin h \phi \cos h t d t .
\end{aligned}
$$

They are homogeneous harmonic polynomials of degree $n$ in $x, y, z$.

We shall be interested in obtaining complete orthogonal systems of harmonic polynomials in the interior of the prolate spheroid

$$
\frac{z^{2}}{\operatorname{ch}^{2} \alpha}+\frac{\rho^{2}}{\operatorname{sh}^{2} \alpha}=1
$$

and in the interior of the oblate spheroid

$$
\frac{z^{2}}{\operatorname{sh}^{2} \alpha}+\frac{\rho^{2}}{\operatorname{ch}^{2} \alpha}=1
$$

Thus it is convenient to introduce coordinates $u, v$ defined by the relations 


$$
z+i \rho=\cos (u-i v)=\cos u \operatorname{ch} v+i \sin u \operatorname{sh} v
$$

for the prolate case, and defined by

$$
\rho+i z=\sin (u+i v)=\sin u \operatorname{ch} v+i \cos u \operatorname{sh} v
$$

for the oblate case. In both cases, the boundaries of the above spheroids have the equation $v=\alpha$.

We define

$$
\begin{aligned}
& U_{n, h}(\rho, z)=\left[\frac{(n+h) !}{(n-h) !}\right]^{1 / 2} \frac{1}{\pi i^{h}} \int_{0}^{\pi} P_{n}(z+i \rho \cos t) \cos h t d t \\
& V_{n, h}(\rho, z)=\left[\frac{(n+h) !}{(n-h) !}\right]^{1 / 2} \frac{i^{n-h}}{\pi} \int_{0}^{\pi} P_{n}(i z-\rho \cos t) \cos h t d t
\end{aligned}
$$

By the addition theorem for the Legendre polynomials we obtain the wellknown expressions

$$
\begin{aligned}
& U_{n, h}(\rho, z)=\left[\frac{(n-h) !}{(n+h) !}\right]^{1 / 2} P_{n}^{h}(\cos u) P_{n}^{h}(\operatorname{ch} v), \\
& V_{n, h}(\rho, z)=\left[\frac{(n-h) !}{(n+h) !}\right]^{1 / 2} i^{n-h} P_{n}^{h}(\cos u) P_{n}^{h}(i \operatorname{sh} v),
\end{aligned}
$$

where in the first case $u, v$ are coordinates in the prolate spheroid (1) and in the second case $u, v$ are coordinates in the oblate spheroid (2).

Here

$$
\begin{gathered}
P_{n}^{h}(\operatorname{ch} v)=\operatorname{sh}^{h} v P_{n}^{(h)}(\operatorname{ch} v), \\
P_{n}^{h}(i \operatorname{sh} v)=\operatorname{ch}^{h} v P_{n}^{(h)}(i \operatorname{sh} v) .
\end{gathered}
$$

The expressions

$$
\begin{array}{ll}
U_{n, h}(\rho, z) \cos h \phi, & U_{n, h}(\rho, z) \sin h \phi, \\
V_{n, h}(\rho, z) \cos h \phi, & V_{n, h}(\rho, z) \sin h \phi
\end{array}
$$

are harmonic polynomials in $x, y, z$ of degree $n$. 
We shall be concerned here with the new polynomials

$$
\begin{aligned}
X_{n, h} & =\frac{\partial}{\partial z} U_{n+1, h} \\
& =\left[\frac{(n+1+h) !}{(n+1-h) !}\right]^{1 / 2} \frac{1}{\pi i^{h}} \int_{0}^{\pi} P_{n+1}^{\prime}(z+i \rho \cos t) \cos h t d t
\end{aligned}
$$

and

$$
\begin{aligned}
Y_{n, h} & =\frac{\partial}{\partial z} V_{n+1, h} \\
& =-\left[\frac{(n+1+h) !}{(n+1-h) !}\right]^{1 / 2} \frac{i^{n-h}}{\pi} \int_{0}^{\pi} P_{n+1}^{\prime}(i z-\rho \cos t) \cos h t d t .
\end{aligned}
$$

The functions

$$
\begin{array}{ll}
X_{n, h}(\rho, z) \cos h \phi, & X_{n, h}(\rho, z) \sin h \phi, \\
Y_{n, h}(\rho, z) \cos h \phi, & Y_{n, h}(\rho, z) \sin h \phi
\end{array}
$$

are linear combinations of the classical spherical harmonics. The functions $X_{n, 0}$ and $Y_{n, 0}$ involve only zonal harmonics and satisfy the partial differential equation

$$
\frac{\partial^{2} f}{\partial \rho^{2}}+\frac{1}{\rho} \frac{\partial f}{\partial \rho}+\frac{\partial^{2} f}{\partial z^{2}}=0
$$

of axially symmetric flow.

Let us denote by $D$ either the prolate or the oblate spheroid described above, and let us denote the Dirichlet integral over $D$ by

$$
\begin{aligned}
(f, g) & =\iiint_{D}\left\{\frac{\partial f}{\partial x} \frac{\partial g}{\partial x}+\frac{\partial f}{\partial y} \frac{\partial g}{\partial y}+\frac{\partial f}{\partial z} \frac{\partial g}{\partial z}\right\} d x d y d z & \\
& =\iint_{S} f \frac{\partial g}{\partial \nu} d \sigma, & (\Delta g=0),
\end{aligned}
$$

where $S$ is the surface of $D$, and where $\nu$ and $d \sigma$ denote outer normal and area 
elements on $S$. Since $z+i \rho=\cos (u-i v)$ and $\rho+i z=\sin (u+i v)$ are isogonal mappings, we obtain, on the spheroid $S$,

$$
d \sigma \frac{\partial}{\partial \nu}=\rho d \phi d u \frac{\partial}{\partial v}
$$

Hence

$$
(f, g)=\iint_{S} f \frac{\partial g}{\partial v} \rho d \phi d u=\int_{0}^{\pi} \int_{0}^{2 \pi} f \frac{\partial g}{\partial v} \rho d \phi d u
$$

3. Orthogonality. If $h \neq k$, we have by the orthogonality of ordinary Fourier series

$$
\begin{aligned}
& \left(U_{n, h} \cos h \phi, \quad U_{m, k} \cos k \phi\right)=0, \\
& \left(U_{n, h} \sin h \phi, \quad U_{m, k} \sin k \phi\right)=0, \\
& \left(U_{n, h} \cos h \phi, \quad U_{m, k} \sin k \phi\right)=0, \\
& \left(U_{n, h} \cos h \phi, \quad U_{m, h} \sin h \phi\right)=0,
\end{aligned}
$$

and similarly for $V_{n, h}$. For $h=k$ we obtain in the prolate spheroid

$$
\begin{gathered}
\left(U_{n, h} \cos h \phi, U_{m, h} \cos h \phi\right)=\int_{0}^{\pi} \int_{0}^{2 \pi} U_{n, h} \frac{\partial U_{m, h}}{\partial v}\left(\cos ^{2} h \phi\right) \rho d \phi d u \\
=\pi\left(1+\delta_{0 h}\right) \frac{(n-h) !}{(n+h) !} P_{n}^{h}(\operatorname{ch} \alpha)\left[\operatorname{sh} \alpha P_{m}^{h+1}(\operatorname{ch} \alpha)+h \operatorname{ch} \alpha P_{m}^{h}(\operatorname{ch} \alpha)\right] \\
\cdot \int_{0}^{\pi} P_{n}^{h}(\cos u) P_{m}^{h}(\cos u) \sin u d u \\
=\frac{2 \pi\left(1+\delta_{0 h}\right)}{2 n+1} P_{n}^{h}(\operatorname{ch} \alpha)\left[\operatorname{sh} \alpha P_{n}^{h+1}(\operatorname{ch} \alpha)+h \operatorname{ch} \alpha P_{n}^{h}(\operatorname{ch} \alpha)\right] \delta_{n m},
\end{gathered}
$$

where $\delta_{n m}=0$ for $n \neq m$ and $\delta_{n n}=1$.

Similarly 


$$
\begin{aligned}
\left(U_{n, h} \sin h \phi,\right. & \left.U_{m, h} \sin h \phi\right) \\
= & \frac{2 \pi}{2 n+1} P_{n}^{h}(\operatorname{ch} \alpha)\left[\operatorname{sh} \alpha P_{n}^{h+1}(\operatorname{ch} \alpha)+h \operatorname{ch} \alpha P_{n}^{h}(\operatorname{ch} \alpha)\right] \delta_{n m} .
\end{aligned}
$$

For the oblate spheroid we have in like manner

$$
\begin{aligned}
\left(V_{n, h} \cos h \phi,\right. & \left.V_{m, h} \cos h \phi\right) \\
= & \pi\left(1+\delta_{0 h}\right) \frac{(n-h) !}{(n+h) !} i^{n+m-2 h} P_{n}^{h}(i \operatorname{sh} \alpha)\left[i \operatorname{ch} \alpha P_{m}^{h+1}(i \operatorname{sh} \alpha)\right. \\
& \left.+h \operatorname{sh} \alpha P_{m}^{h}(i \operatorname{sh} \alpha)\right] \int_{0}^{\pi} P_{n}^{h}(\cos u) P_{m}^{h}(\cos u) \sin u d u \\
= & \frac{2 \pi\left(1+\delta_{0 h}\right)}{2 n+1}(-1)^{n-h} P_{n}^{h}(i \operatorname{sh} \alpha)\left[i \operatorname{ch} \alpha P_{n}^{h+1}(i \operatorname{sh} \alpha)\right. \\
& \left.+h \operatorname{sh} \alpha P_{n}^{h}(i \operatorname{sh} \alpha)\right] \delta_{n m}
\end{aligned}
$$

Also

$\left(V_{n, h} \sin h \phi, V_{m, h} \sin h \phi\right)$

$$
=\frac{2 \pi}{2 n+1}(-1)^{n-h} P_{n}^{h}(i \operatorname{sh} \alpha)\left[i \operatorname{ch} \alpha P_{n}^{h+1}(i \operatorname{sh} \alpha)+h \operatorname{sh} \alpha P_{n}^{h}(i \operatorname{sh} \alpha)\right] \delta_{n m} .
$$

We have therefore proved:

THEOREM 1. The harmonic polynomials $U_{n, h} \cos h \phi, U_{n, h} \sin h \phi$ form a complete orthogonal system for the interior of the prolate spheroid (1) in the sense of the Dirichlet integral. The harmonic polynomials $V_{n, h} \cos h \phi, V_{n, h}$ $\sin h \phi$ form a similar system inside the oblate spheroid (2). The polynomials $U_{n, 0}$ and $V_{n, 0}$ alone form, respectively, complete orthogonal systems for the equation of axially symmetric flow inside the spheroids (1) and (2).

We turn next to a less obvious result for the polynomials $X_{n, h}$ and $Y_{n, h}$. Let

$$
[f, g]=\iiint_{D} f g d x d y d z
$$


Then clearly, if $h \neq k$,

$$
\begin{aligned}
& {\left[X_{n, h} \cos h \phi, X_{m, k} \cos k \phi\right]=0,} \\
& {\left[X_{n, h} \sin h \phi, X_{m, k} \sin k \phi \gamma=0,\right.} \\
& {\left[X_{n, h} \cos h \phi, X_{m, k} \sin k \phi\right]=0,} \\
& {\left[X_{n, h} \cos h \phi, X_{m, h} \sin h \phi\right]=0,}
\end{aligned}
$$

and similarly for $Y_{n, h}$. Now

$$
\frac{\partial}{\partial z}=\frac{\partial u}{\partial z} \frac{\partial}{\partial u}+\frac{\partial v}{\partial z} \frac{\partial}{\partial v}
$$

when $z+i \rho=\cos (u-i v)$. Also

$$
\begin{aligned}
\frac{\partial u}{\partial z}-i \frac{\partial v}{\partial z}=\frac{d(u-i v)}{d(z+i \rho)}=\frac{d(z-i \rho)}{d(u+i v)} \frac{d(u+i v)}{d(z-i \rho)} & \frac{d(u-i v)}{d(z+i \rho)} \\
& =-\frac{\partial(u, v)}{\partial(z, \rho)} \sin (u+i v) .
\end{aligned}
$$

Therefore

$\left[X_{n, h} \cos h \phi, f\right]$

$$
\begin{gathered}
=-\iiint_{D} f \cosh \phi\left\{\frac{\partial U_{n+1, h}}{\partial u} \sin u \operatorname{ch} v-\frac{\partial U_{n+1, h}}{\partial v} \cos u \operatorname{sh} v\right\} \\
\cdot \frac{\partial(u, v)}{\partial(z, \rho)} \rho d \phi d \rho d z \\
=\left[\frac{(n+1-h) !}{(n+1+h) !}\right]_{0}^{1 / 2} \int_{0}^{a} \int_{0}^{2 \pi} f \cos h \phi \sin u \operatorname{sh} v \\
\left.+P_{n+1}^{h}(\cos u) P_{n+1}^{h+1}(\operatorname{ch} v) \cos u \operatorname{sh} v\right] d \phi d u d v .
\end{gathered}
$$

The last integral vanishes when $f$ is a harmonic polynomial of the form 


$$
P_{m}^{h}(\cos u) P_{m}^{h}(\operatorname{ch} v) \cos h \phi
$$

with $m<n$, since

$$
\begin{aligned}
& \int_{0}^{\pi} P_{n+1}^{h+1}(\cos u) \sin u P_{m}^{h}(\cos u) \sin u d u=0, \\
& \int_{0}^{\pi} P_{n+1}^{h}(\cos u) \cos u P_{m}^{h}(\cos u) \sin u d u=0
\end{aligned}
$$

Hence for $n \neq m$

$$
\left[X_{n, h} \cos h \phi, X_{m, h} \cos h \phi\right]=0,
$$

and similarly

$$
\left[X_{n, h} \sin h \phi, X_{m, h} \sin h \phi\right]=0 \text {. }
$$

For $m=n$, we have

$$
f=X_{n, h} \cos h \phi=\left[\frac{n+1+h}{n+1-h}\right]^{1 / 2}(2 n+1) U_{n, h} \cos h \phi+\cdots,
$$

where the dots indicate harmonic polynomials of lower degree, which are orthogonal to $X_{n, h} \cos h \phi$. Thus

$$
\begin{aligned}
& {\left[X_{n, h} \cos h \phi, X_{n, h} \cos h \phi\right]} \\
& \begin{array}{r}
=(2 n+1) \frac{(n-h) !}{(n+h) !} \int_{0}^{a} \int_{0}^{\pi} \int_{0}^{2 \pi} \cos ^{2} h \phi \sin u \operatorname{sh} v P_{n}^{h}(\cos u) P_{n}^{h}(\operatorname{ch} v) \\
\quad \cdot\left[P_{n+1}^{h+1}(\cos u) P_{n+1}^{h}(\operatorname{ch} v) \sin u \operatorname{ch} v\right. \\
\left.\quad+P_{n+1}^{h}(\cos u) P_{n+1}^{h+1}(\operatorname{ch} v) \cos u \operatorname{sh} v\right] d \phi d u d v \\
=\pi\left(1+\delta_{0 h}\right) \frac{(n-h) !}{(n+h) !} \int_{0}^{a} \int_{0}^{\pi} P_{n+1}^{h+1}(\cos u)^{2} P_{n}^{h}(\operatorname{ch} v) \\
P_{n+1}^{h}(\operatorname{ch} v) \operatorname{sh} v \operatorname{ch} v \sin u d u d v
\end{array}
\end{aligned}
$$


$+\pi\left(1+\delta_{0 h}\right) \frac{(n-h+1) !}{(n+h) !} \int_{0}^{\alpha} \int_{0}^{\pi} P_{n+1}^{h}(\cos u)^{2} P_{n}^{h}(\operatorname{ch} v)$

$P_{n+1}^{h+1}(\operatorname{ch} v) \operatorname{sh}^{2} v \sin u d u d v$

$=\frac{2 \pi\left(1+\delta_{0 h}\right)}{2 n+3}(n+1+h) \int_{0}^{a} P_{n}^{h}(\operatorname{ch} v) \operatorname{sh} v$

$\cdot\left[(n+2+h) P_{n+1}^{h}(\operatorname{ch} v) \operatorname{ch} v+P_{n+1}^{h+1}(\operatorname{ch} v) \operatorname{sh} v\right] d v$.

The same value is obtained if we replace $\cos h \phi$ by $\sin h \phi$ throughout, $h>0$.

For the oblate spheroids, we have, on the other hand,

$$
\frac{\partial}{\partial z}=\frac{\partial u}{\partial z} \frac{\partial}{\partial u}+\frac{\partial v}{\partial z} \frac{\partial}{\partial v}
$$

with $\rho+i z=\sin (u+i v)$. Hence

$$
\begin{aligned}
\frac{\partial u}{\partial z}-i \frac{\partial v}{\partial z}=\frac{d(u-i v)}{d(z+i \rho)} & =\frac{d(\rho+i z)}{d(u+i v)} \frac{d(u+i v)}{d(\rho+i z)} \frac{d(u-i v)}{d(z+i \rho)} \\
& =-i \frac{\partial(u, v)}{\partial(\rho, z)} \cos (u+i v) .
\end{aligned}
$$

Therefore

$\left[Y_{n, h} \cos h \phi, f\right]$

$$
\begin{gathered}
=-\iiint_{D} f \cos h \phi\left\{\frac{\partial V_{n+1, h}}{\partial u} \sin u \operatorname{sh} v-\frac{\partial V_{n+1, h}}{\partial v} \cos u \operatorname{ch} v\right\} \\
\cdot \frac{\partial(u, v)}{\partial(\rho, z)} \rho d \phi d \rho d z \\
=i^{n+1-h}\left[\frac{(n+1-h) !}{(n+1+h) !}\right]^{1 / 2} \int_{0}^{a} \int_{0}^{\pi} \int_{0}^{2 \pi} f \cos h \phi \sin u \operatorname{ch} v \\
\cdot\left[P_{n+1}^{h}(i \operatorname{sh} v) P_{n+1}^{h+1}(\cos u) \sin u \operatorname{sh} v\right.
\end{gathered}
$$




$$
\left.+i P_{n+1}^{h}(\cos u) P_{n+1}^{h+1}(i \operatorname{sh} v) \cos u \operatorname{ch} v\right] d \phi d u d u
$$

This integral vanishes when $f$ is a harmonic polynomial

$$
P_{m}^{h}(\cos u) P_{m}^{h}(i \operatorname{sh} v) \cos h \phi
$$

of degree $m<n$, since

$$
\begin{aligned}
& \int_{0}^{\pi} P_{n+1}^{h+1}(\cos u) \sin u P_{m}^{h}(\cos u) \sin u d u=0, \\
& \int_{0}^{\pi} P_{n+1}^{h}(\cos u) \cos u P_{m}^{h}(\cos u) \sin u d u=0 .
\end{aligned}
$$

Hence, for $n \neq m$,

$$
\left[Y_{n, h} \cos h \phi, Y_{m, h} \cos h \phi\right]=0,
$$

and also

$$
\left[Y_{n, h} \sin h \phi, \quad Y_{m, h} \sin h \phi\right]=0
$$

For $m=n$, we note that

$$
f=Y_{n, h} \cos h \phi=-\left[\frac{n+1+h}{n+1-h}\right]^{1 / 2}(2 n+1) V_{n, h} \cos h \phi+\cdots,
$$

where the dots represent harmonic polynomials of lower degree, which are orthogonal to $Y_{n, h} \cos h \phi$. Therefore

$\left[Y_{n, h} \cos h \phi, Y_{n, h} \cos h \phi\right]$

$$
\begin{gathered}
=-(2 n+1) i^{2 n-2 h+1} \frac{(n-h) !}{(n+h) !} \int_{0}^{\alpha} \int_{0}^{\pi} \int_{0}^{2 \pi} \cos ^{2} h \phi \sin u \operatorname{ch} v \\
P_{n}^{h}(\cos u) P_{n}^{h}(i \operatorname{sh} v) \cdot\left[P_{n+1}^{h}(i \operatorname{sh} v) P_{n+1}^{h+1}(\cos u) \sin u \operatorname{sh} v\right. \\
\left.+i P_{n+1}^{h}(\cos u) P_{n+1}^{h+1}(i \operatorname{sh} v) \cos u \operatorname{ch} v\right] d \phi d u d v
\end{gathered}
$$




$$
\begin{aligned}
& =\frac{2 \pi\left(1+\delta_{0 h}\right)}{2 n+3}(-1)^{n-h+1} i(n+1+h) \int_{0}^{a} P_{n}^{h}(i \operatorname{sh} v) \operatorname{ch} v \\
& \cdot\left[(n+2+h) P_{n+1}^{h}(i \operatorname{sh} v) \operatorname{sh} v+i P_{n+1}^{h+1}(i \operatorname{sh} v) \operatorname{ch} v\right] d v .
\end{aligned}
$$

W'e obtain the same value if $\cos h \phi$ is replaced by $\sin h \phi$.

This completes the proof of:

THEOREM 2. The harmonic polynomials $X_{n, h} \cos h \phi, X_{n, h} \sin h \phi$ form a complete orthogonal system for the interior of the prolate spheroid (1) in the sense of the scalar product

$$
[f, g]=\iiint_{D} f g d x d y d z .
$$

The corresponding system in the oblate spheroid (2) is

$$
Y_{n, h} \cos h \phi, Y_{n, h} \sin h \phi
$$

The zonal polynomials $X_{n, 0}$ and $Y_{n, 0}$ are complete and orthogonal for the equation of axially symmetric flow in their respective domains (1) and (2).

Friedrichs [4] has investigated the eigenvalue problem

$$
\frac{\left[f_{z}, f_{z}\right]}{(f, f)}=\frac{\iiint_{D}(\partial f / \partial z)^{2} d x d y d z}{\iiint_{D}\left\{(\partial f / \partial x)^{2}+(\partial f / \partial y)^{2}+(\partial f / \partial z)^{2}\right\} d x d y d z}=\text { maximum }
$$

for harmonic functions $f$ in quite general regions $D$ of space. It is clear from Theorem 1 and Theorem 2 that we have:

THEOREM 3. The eigenfunctions for the problem

$$
\frac{\left[f_{z}, f_{z}\right]}{(f, f)}=\text { maximum, } \Delta f=0 \text {, }
$$

in the prolate spheroid (1) are

$$
U_{n, h} \cos h \phi, \quad U_{n, h} \sin h \phi,
$$

and in the oblate spheroid (2) they are 


$$
V_{n, h} \cos h \phi, V_{n, h} \sin h \phi .
$$

The corresponding eigenvalues are

$$
(n+1+h) \frac{\int_{0}^{\alpha} P_{n}^{h}(\operatorname{ch} v) \operatorname{sh} v\left[(n+2+h) P_{n+1}^{h}(\operatorname{ch} v) \operatorname{ch} v+P_{n+1}^{h+1}(\operatorname{ch} v) \operatorname{sh} v\right] d v}{P_{n+1}^{h}(\operatorname{ch} \alpha)\left[\operatorname{sh} \alpha P_{n+1}^{h+1}(\operatorname{ch} \alpha)+h \operatorname{ch~} \alpha P_{n+1}^{h}(\operatorname{ch} \alpha)\right]}
$$

for the prolate spheroids and $(n+1+h) Q$, where $Q$ is the expression

$$
\frac{i \int_{0}^{a} P_{n}^{h}(i \operatorname{sh} v) \operatorname{ch} v\left[(n+2+h) P_{n+1}^{h}(i \operatorname{sh} v) \operatorname{sh} v+i P_{n+1}^{h+1}(i \operatorname{sh} v) \operatorname{ch} v\right] d v}{P_{n+1}^{h}(i \operatorname{sh} \alpha)\left[i \operatorname{ch} \alpha P_{n+1}^{h+1}(i \operatorname{sh} \alpha)+h \operatorname{sh} \alpha P_{n+1}^{h}(i \operatorname{sh} \alpha)\right]},
$$

for the oblate spheroids.

Friedrichs was led to this extremal problem through his investigation of Korn's inequality and existence theorems for the partial differential equations of elasticity. We shall show in the following how the eigenfunctions can be used to solve the biharmonic equation.

One sees easily from Theorem 3 that

$$
U_{n, h} \cos h \phi, U_{n, h} \sin h \phi
$$

and

$$
V_{n, h} \cos h \phi, V_{n, h} \sin h \phi
$$

are also orthogonal in the norm

$$
\iiint_{D}\left\{\left(\frac{\partial f}{\partial x}\right)^{2}+\left(\frac{\partial f}{\partial x}\right)^{2}\right\} d x d y d z=(f, f)-\left[f_{z}, f_{z}\right]
$$

However, we do not go into details since this norm leads to no apparent application.

One can obtain quite interesting results, on the other hand, by using the orthogonality of the $X_{n, h}$ and the $Y_{n, h}$ over the interior of the ellipses $(1)$ and 
(2) for all values of $\alpha$ to obtain a corresponding orthogonality of the same polynomials over the surface of the spheroids with respect to a suitable weight function. Indeed, we have

$\frac{d}{d \alpha}\left[X_{n, h} \cos h \phi, X_{m, k} \cos k \phi\right]$

$$
\begin{gathered}
=\frac{2 \pi\left(1+\delta_{0 h}\right)(n+1+h) \delta_{h k} \delta_{n m}}{2 n+3} \frac{d}{d \alpha} \int_{0}^{\alpha} P_{n}^{h}(\operatorname{ch} v) \operatorname{sh} v \\
\cdot\left[(n+2+h) P_{n+1}^{h}(\operatorname{ch} v) \operatorname{ch} v+P_{n+1}^{h+1}(\operatorname{ch} v) \operatorname{sh} v\right] d v,
\end{gathered}
$$

whence

$$
\begin{aligned}
\iint_{S}\left\{X_{n, h} \cos h \phi X_{m, k} \cos k \phi\right\}\left|1-(z+i \rho)^{2}\right|^{1 / 2} d \sigma \\
=\frac{2 \pi\left(1+\delta_{0 h}\right)(n+h+1)}{2 n+3} P_{n}^{h}(\operatorname{ch} \alpha) \operatorname{sh} \alpha \\
\cdot\left[(n+2+h) P_{n+1}^{h}(\operatorname{ch} \alpha) \operatorname{ch} \alpha+P_{n+1}^{h+1}(\operatorname{ch} \alpha) \operatorname{sh} \alpha\right] \delta_{h k} \delta_{n m} .
\end{aligned}
$$

Likewise, by the same reasoning,

$$
\begin{aligned}
\iint_{S}\left\{Y_{n, h} \cos h \phi Y_{m, k} \cos k \phi\right\}\left|1-(\rho+i z)^{2}\right|^{1 / 2} d \sigma \\
=\frac{2 \pi\left(1+\delta_{0 h}\right)(n+h+1)}{2 n+3} i(-1)^{n-h+1} P_{n}^{h}(i \operatorname{sh} \alpha) \operatorname{ch} \alpha \\
\cdot\left[(n+2+h) P_{n+1}^{h}(i \operatorname{sh} \alpha) \operatorname{sh} \alpha+i P_{n+1}^{n+1}(i \operatorname{sh} \alpha) \operatorname{ch} \alpha\right] \delta_{h k} \delta_{n m},
\end{aligned}
$$

with exactly the same formulas in both cases if $\cos h \phi$ is replaced by $\sin h \phi$.

This calculation yields:

THEOREM 4. The polynomials $X_{n, h} \cos h \phi, X_{n, h} \sin h \phi$ are complete and orthogonal over the surface of the spheroid (1) in the sense of the scalar product 


$$
\{f, g\}=\iint_{S} f g\left|1-(z+i \rho)^{2}\right|^{1 / 2} d \sigma
$$

with weight function $|1-(z+i \rho)|^{1 / 2}$ equal to the square root of the product of the distances from $(\rho, \phi, z)$ to the points $(0,0,1)$ and $(0,0,-1)$. The harmonic polynomials $Y_{n, h} \cos h \phi, Y_{n, h}$ sin $h \phi$ are complete and orthogonal over the surface of the oblate spheroid (2) in the sense of the scalar product

$$
\{f, g\}=\iint_{S} f g\left|1-(\rho+i z)^{2}\right|^{1 / 2} d \sigma .
$$

There exist quite clearly further orthogonality properties of the polynomials $U_{n, h}$ and $V_{n, h}$ which do not depend on the shape of the spheroids (1) and (2). However, we make no pretense here at tabulating all possible orthogonal harmonic polynomials of this type ( $\mathrm{cf} .[8]$ ), but proceed rather to apply the results already obtained to the Laplace and biharmonic equations.

4. The kernels. The Green's function $G(P, Q)$ for the Laplace equation in a region $D$ is a harmonic function of the coordinates $x, y, z$ of the point $P$ in $D$, except at $Q$, where

$$
G(P, Q)=\frac{1}{r(P, Q)}+\text { harmonic terms },
$$

and it vanishes for $P$ on the surface $S$ of $D$. Here $r(P, Q)$ denotes the distance from $P$ to $Q$. The Neumann's function $N(P, Q)$ has a similar fundamental singularity,

$$
N(P, Q)=\frac{1}{r(P, Q)}+\text { harmonic terms }
$$

while its normal derivative is constant on $S$ and

$$
\iint_{S} N(P, Q) d \sigma(P)=0 .
$$

The harmonic kernel function $K(P, Q)$ is defined by the formula [2]

$$
K(P, Q)=\frac{1}{4 \pi}\{N(P, Q)-G(P, Q)\} .
$$


If $f_{n}(P)$ is a complete orthonormal system of harmonic functions in $D$ in the sense

$$
\left(f_{n}, f_{m}\right)=\delta_{n m},
$$

with

$$
\iint_{S} f d \sigma=0
$$

then one has the Bergman expansion

$$
K(P, Q)=\sum_{n=1}^{\infty} f_{n}(P) f_{n}(Q) .
$$

On the other hand, if $g_{n}(P)$ is a complete orthonormal system of harmonic functions in $D$ in the sense of the scalar product

$$
\{f, g\}=\iint_{S} f g \omega d \sigma
$$

corresponding to an arbitrary positive weight function $\omega$ on $S$, then the kernel

$$
H(P, Q)=\sum_{n=1}^{\infty} g_{n}(P) g_{n}(Q)
$$

is given by [7]

$$
H(P, Q)=\frac{1}{(4 \pi)^{2}} \iint_{S} \frac{1}{\omega(T)} \frac{\partial G(T, P)}{\partial \nu(T)} \frac{\partial G(T, Q)}{\partial \nu(T)} d \sigma(T) .
$$

For $P$ on $S$ we have

$$
\omega(P) H(P, Q)=-\frac{1}{4 \pi} \frac{\partial G(P, Q)}{\partial \nu(P)} .
$$

The Green's function $\Gamma(P, Q)$ of the biharmonic equation

$$
\Delta \Delta F=0
$$

is a biharmonic function of the coordinates of $P$, except at $Q$, where 


$$
\Gamma(P, Q)=-r(P, Q)+\text { biharmonic terms },
$$

and for $P$ on $S$ it satisfies

$$
\Gamma(P, Q)=\frac{\partial \Gamma(P, Q)}{\partial \nu(P)}=0
$$

If $h_{n}(P)$ is a complete orthonormal system of harmonic functions in the sense

$$
\left[h_{n}, h_{m}\right]=\delta_{n m},
$$

then the kernel function

$$
k(P, Q)=\sum_{n=1}^{\infty} h_{n}(P) h_{n}(Q)
$$

is given by the identity $[5,10]$

$$
k(P, Q)=-\frac{1}{8 \pi} \Delta(P) \Delta(Q) \Gamma(P, Q) .
$$

The relation here between the harmonic functions $h_{n}$ and the biharmonic kernel function $k$ is a consequence of the nature of the energy integral

$$
\iiint_{D}(\Delta F)^{2} d x d y d z
$$

for the biharmonic equation.

We discuss here the expansion of the kernels $K, H$, and $k$ in terms of the orthogonal polynomials of $\xi 3$ for the case where $D$ is a prolate or oblate spheroid. One obtains easily from The orems 1, 2, and 4, together with the computation of the related normalization constants, the following results:

THEOREM 5. In the prolate spheroid (1) we have

$K\left(\rho, z, \phi ; \rho^{\prime}, z^{\prime}, \phi^{\prime}\right)$

$=\sum_{n=1}^{\infty} \sum_{h=0}^{n} \frac{(2 n+1) U_{n, h}(\rho, z) U_{n, h}\left(\rho^{\prime}, z^{\prime}\right) \cos h\left(\phi-\phi^{\prime}\right)}{2 \pi\left(1+\delta_{0 h}\right) P_{n}^{h}(\operatorname{ch} \alpha)\left[\operatorname{sh} \alpha P_{n}^{h+1}(\operatorname{ch} \alpha)+h \operatorname{ch} \alpha P_{n}^{h}(\operatorname{ch} \alpha)\right]}+C$, 
where $C$ is a constant chosen to agree with the normalization of Neumann's function. In the oblate spheroid (2),

$$
\begin{aligned}
K\left(\rho, z, \phi ; \rho^{\prime}, z^{\prime}, \phi^{\prime}\right)= & \sum_{n=1}^{\infty} \sum_{h=0}^{n} \frac{(-1)^{n-h}(2 n+1)}{2 \pi\left(1+\delta_{0 h}\right)} \\
& \frac{V_{n, h}(\rho, z) V_{n, h}\left(\rho^{\prime}, z^{\prime}\right) \cos h\left(\phi-\phi^{\prime}\right)}{P_{n}^{h}(i \operatorname{sh} \alpha)\left[i \operatorname{ch} \alpha P_{n}^{h+1}(i \operatorname{sh} \alpha)+h \operatorname{sh} \alpha P_{n}^{h}(i \operatorname{sh} \alpha)\right]}+C,
\end{aligned}
$$

where again $C$ is a suitable constant.

THEOREM 6. In the prolate spheroid (1),

$$
\begin{aligned}
k\left(\rho, z, \phi ; \rho^{\prime}, z^{\prime}, \phi^{\prime}\right)=\sum_{n=0}^{\infty} \sum_{h=0}^{n} \frac{(2 n+3)}{2 \pi\left(1+\delta_{0 h}\right)(n+1+h)} \\
\frac{X_{n, h}(\rho, z) X_{n, h}\left(\rho^{\prime}, z^{\prime}\right) \cos h\left(\phi-\phi^{\prime}\right)}{\int_{0}^{a} P_{n}^{h}(\operatorname{ch} v) \operatorname{sh} v\left[(n+2+h) P_{n+1}^{h}(\operatorname{ch} v) \operatorname{ch} v+P_{n+1}^{h+1}(\operatorname{ch} v) \operatorname{sh} v\right] d v} .
\end{aligned}
$$

In the oblate spheroid (2),

$$
\begin{aligned}
& k\left(\rho, z, \phi ; \rho^{\prime}, z^{\prime}, \phi^{\prime}\right)=\sum_{n=0}^{\infty} \sum_{h=0}^{n} \frac{(-1)^{n-h} i(2 n+3)}{2 \pi\left(1+\delta_{0 h}\right)(n+1+h)} \\
& \frac{Y_{n, h}(\rho, z) Y_{n, h}\left(\rho^{\prime}, z^{\prime}\right) \cos h\left(\phi-\phi^{\prime}\right)}{\int_{0}^{a} P_{n}^{h}(i \operatorname{sh} v) \operatorname{ch} v\left[(n+2+h) P_{n+1}^{h}(i \operatorname{sh} v) \operatorname{sh} v+i P_{n+1}^{h+1}(i \operatorname{sh} v) \operatorname{ch} v\right] d v} .
\end{aligned}
$$

THEOREM 7. In the prolate spheroid (1),

$$
\begin{aligned}
H\left(\rho, z, \phi ; \rho^{\prime}, z^{\prime}, \phi^{\prime}\right) & =\sum_{n=0}^{\infty} \sum_{h=0}^{n} \frac{(2 n+3)}{2 \pi\left(1+\delta_{0 h}\right)(n+1+h)} \\
& \frac{X_{n, h}(\rho, z) X_{n, h}\left(\rho^{\prime}, z^{\prime}\right) \cos h\left(\phi-\phi^{\prime}\right)}{P_{n}^{h}(\operatorname{ch} \alpha) \operatorname{sh} \alpha\left[(n+2+h) P_{n+1}^{h}(\operatorname{ch} \alpha) \operatorname{ch} \alpha+P_{n+1}^{h+1}(\operatorname{ch} \alpha) \operatorname{sh} \alpha\right]},
\end{aligned}
$$

when $\omega=\left|1-(z+i \rho)^{2}\right|^{1 / 2}$. If $\omega=\left|1-(\rho+i z)^{2}\right|^{1 / 2}$, we have, for the oblate spheroid (2), 


$$
\begin{aligned}
H\left(\rho, z, \phi ; \rho^{\prime}, z^{\prime}, \phi^{\prime}\right) & =\sum_{n=0}^{\infty} \sum_{h=0}^{n} \frac{(2 n+3) i(-1)^{n-h}}{2 \pi\left(1+\delta_{0 h}\right)(n+1+h)} \\
& \frac{Y_{n, h}(\rho, z) Y_{n, h}\left(\rho^{\prime}, z^{\prime}\right) \cos h\left(\phi-\phi^{\prime}\right)}{P_{n}^{h}(i \operatorname{sh} \alpha) \operatorname{ch} \alpha\left[(n+2+h) P_{n+1}^{h}(i \operatorname{sh} \alpha) \operatorname{sh} \alpha+i P_{n+1}^{h+1}(i \operatorname{sh} \alpha) \operatorname{ch} \alpha\right]}
\end{aligned}
$$

Theorem 7 is of interest because it yields, say for (1), the relation

$$
\begin{array}{r}
-\frac{1}{4 \pi} \frac{\partial G\left(\rho, z, \phi ; \rho^{\prime}, z^{\prime}, \phi^{\prime}\right)}{\partial \nu}=\left|1-(z+i \rho)^{2}\right|^{1 / 2} \sum_{n=0}^{\infty} \sum_{h=0}^{n} \frac{(2 n+3)}{2 \pi\left(1+\delta_{0 h}\right)(n+1+h)} \\
\frac{X_{n, h}(\rho, z) X_{n, h}\left(\rho^{\prime}, z^{\prime}\right) \cos h\left(\phi-\phi^{\prime}\right)}{P_{n}^{h}(\operatorname{ch} \alpha) \operatorname{sh} \alpha\left[(n+2+h) P_{n+1}^{h}(\operatorname{ch} \alpha) \operatorname{ch} \alpha+P_{n+1}^{h+1}(\operatorname{ch} \alpha) \operatorname{sh} \alpha\right]}
\end{array}
$$

when the point $\rho, z, \phi$ lies on $S$. This formula can be compared with the corresponding, more classical, formula which follows from Theorem 5.

Theorem 6 permits one to calculate the biharmonic Green's function for prolate or oblate spheroids, and thus in turn to solve the biharmonic boundaryvalue problem in this case. Indeed, we have (cf. [5])

$$
\Gamma(P, Q)=\frac{1}{2 \pi} \iiint_{D} \frac{d \sigma(T)}{r(T, P) r(T, Q)}-\frac{1}{2 \pi} \iiint_{D} \iiint_{D} \frac{k(T, R) d \sigma(T) d \sigma(R)}{r(T, P) r(R, Q)} .
$$

It is significant to note in this connection that all our results can be extended to the case of the region outside a spheroid. One has merely to replace for this purpose the Legendre functions $P_{n}^{h}$ by the Legendre functions $Q_{n}^{h}$ of second kind [6]. Thus $U_{n, h}$ should be replaced, for example, by

$$
\int_{0}^{\pi} Q_{n}(z+i \rho \cos t) \cos h t d t
$$

and $\ddot{V}_{n, h}$ should be replaced by

$$
\int_{0}^{\pi} Q_{n}(i z-\rho \cos t) \cos h t d t
$$

Finally, by combining both kinds of functions, one can obtain orthonormal systems in the region between two confocal spheroids. Thus one might develop 
elaborate formulas for the solution of the biharmonic equation in such shell regions using the basic method of this paper.

\section{REFERENCES}

1. S. Bergman, The kernel function and conformal mapping, Mathematical Surveys, No. 5, American Mathematical Society, New York, 1950.

2. S. Bergman and M. Schiffer, A representation of Green's and Neumann's functions in the theory of partial differential equations of second order, Duke Nath. J. 14 (1947), 609-630.

3. K. O. Friedrichs, On certain inequalities and characteristic value problems for analytic functions and for functions of two variables, Trans. Amer. Math. Soc. 41 (1937), 321 - 364.

4. $581-592$. ,An inequality for potential functions, Amer. J. Math. 68 (1946),

5. P. R. Garabedian, $A$ partial differential equation arising in conformal mapping, Pacific J. Math., 1 (1951), 485 - 524. 1931.

6. E. W. Hobson, The theory of spherical and ellipsoidal harmonics, Cambridge,

7. M. Schiffer, On various types of orthogonalization, Duke Math. J. 17 (1950), $329-366$.

8. G. Szegö, A problem concerning orthogonal polynomials, Trans. Amer. Math. Soc. 37 (1935), 196- 206.

9. New York, 1939.

Orthogonal Polynomials, Amer. Math. Soc. Colloq. Publ., Vol. 23,

10. S. Zaremba, L'équation biharmonique et une classe remarquable de fonctions fondamentales harmoniques, Bull. Int. Acad. Polon. Sci Lett. Cl. Sci. Math. Nat. Sér. A. Sci. Math., 1907, pp. $147-196$.

STANFORD UNIVERSITY 



\section{PACIFIC JOURNAL OF MATHEMATICS}

\section{EDITORS}

\author{
R. M. BOEINSON \\ University of California \\ Berkeley 4, California \\ E. HewitT \\ University of Washington \\ Seattle 5 , Washington
}

\section{R. P. DILWOR TH}

California Institute of Technology

Pasadena 4, California

E. F. BECKENBACH

University of California

Los Angeles 24, California

\section{ASSOCIATE EDITORS}

$\begin{array}{ll}\text { H. BUSEMANN } & \text { P. R. HALMOS } \\ \text { HERBERT FFDERER } & \text { IIEINZ HOPF } \\ \text { MARSHALL IJALL } & \text { R. D. JAMES }\end{array}$

\author{
BøRGE JESSEN \\ PAUL LÉVY \\ GEORGE PÓLYA
}

\author{
J. J. STOKER \\ E. G. STR AUS \\ KÔSAKU YOSIDA
}

\section{SFONSORS}

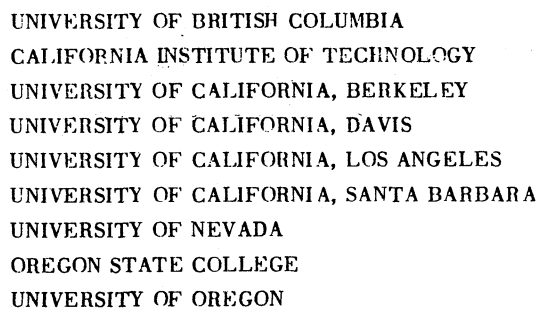

\author{
UNIVERSITY OF SOUTHERN CALIFORNIA \\ STANFORD RESEARCH INSTITUTE \\ STANFORD UNIVERSITY \\ WASHINGTON STATE COLLEGE \\ UNIVERSITY OF WASHINGTON \\ AMERICAN MATHEMATICAL SOCIETY \\ NATIONAL BUREAU OF STANDARDS, \\ INSTITUTE FOR NUMERICAL ANALYSIS
}

Mathematical papers intended for publication in the Pacific Journal of Mathematics should be typewritten (double spaced), and the author should keep a complete copy. Manuscripts may be sent to any of the editors except Robinson, whose term expires with the completion of the present volume; they might also be sent to M.M. Schiffer, Stanford University, Stanford, California, who is succeeding Robinson. All other communications to the editors should be addressed to the managing editor, E. F. Beckenbach, at the address given above.

Authors are entitled to receive 100 free reprints of their published papers and may obtain additional copies at cost.

The Pacific Journal of Mathematics is published quarterly, in March, June, September, and December. The price per volume (4 numbers) is $\$ 8.00$; single issues, $\$ 2.50$. Special price to individual faculty members of supporting institutions and to individual members of the American Mathematical Society: $\$ 4.00$ per volume; single issues, $\$ 1.25$.

Subscriptions, orders for back numbers, and changes of address should be sent to the publishers, University of California Press, Berkeley 4, California.

Printed at Ann Arbor, Michigan. Entered as second class matter at the Post Office, Berkeley, California.

\section{UNIVERSITY OF CALIFORNIA PRESS • BERKELEY AND LOS ANGELES}




\section{Pacific Journal of Mathematics}

\section{Vol. 3, No. 3 \\ May, 1953}

L. Carlitz, Some theorems on generalized Dedekind sums ............ 513

L. Carlitz, The reciprocity theorem for Dedekind sums ............. 523

Edward Richard Fadell, Identifications in singular homology theory..... . . 529

Harley M. Flanders, A method of general linear frames in Riemannian

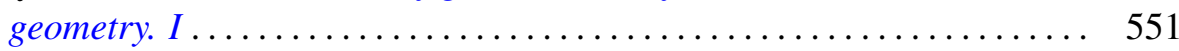

Watson Bryan Fulks, The Neumann problem for the heat equation ........ 567

Paul R. Garabedian, Orthogonal harmonic polynomials.............. 585

R. E. Greenwood and Andrew Mattei Gleason, Distribution of round-off errors for running averages .............................. 605

Arthur Eugene Livingston, The space $H^{p}, 0<p<1$, is not normable ... 613

M. N. Mikhail, On the order of the reciprocal set of a basic set of polynomials .......................................... 617

Louis Joel Mordell, On the linear independence of algebraic numbers . . . . 625

Leo Sario, Alternating method on arbitrary Riemann surfaces .......... 631

Harold Nathaniel Shapiro, Iterates of arithmetic functions and a property of the sequence of primes.............................. 647

H. Shniad, Convexity properties of integral means of analytic functions . . . . 657

Marlow C. Sholander, Plane geometries from convex plates ........... 667 